\title{
Chapter 5 \\ Existence theorems on convolution of functions, distributions and ultradistributions
}

\author{
ANDRZEJ KAMIŃSKI, SVETLANA MINCHEVA-KAMIŃSKA
}

2010 Mathematics Subject Classification: Primary: 44A35, Secondary: 46F05, 46F10.

Key words and phrases: convolution of functions, convolution of (tempered) distributions, convolution of Beurling (tempered) ultradistributions, compatible sets, polynomially compatible sets, $M$-compatible sets.

\subsection{Introduction}

There are known sufficient conditions for existence of convolution in various spaces of functions and generalized functions. They are often given in the form of suitable assumptions concerning the growth of generalized functions.

There exist also conditions of another type, formulated without any restriction on the growth, but expressed in terms of supports of generalized functions and called compatibility conditions (see e.g. [1], p. 124-127). We discuss this notion in some spaces of functions, distributions and ultradistributions and present theorems on existence of the convolution assuming compatibility of supports of the considered functions or generalized functions.

As a matter of fact, we will present conditions of compatibility which are not only sufficient for existence of convolution in the considered spaces of generalized functions, but also necessary in some sense (namely, in the sense of S.Yu. Prishtshepionok who posed in 1977 certain problems concerning the convolution in $\mathcal{D}^{\prime}$ and in $\mathcal{S}^{\prime}$ ); for suitable results we refer to [10], [11], [15]. 
We will show certain new situations, in which the convolution of generalized functions exists, delivering interesting cases of compatibility of supports. In particular, we show that there is a variety of sets in $\mathbb{R}^{d}$, that we call spiral, such that functions, distributions or ultradistributions having supports contained in such sets are convolvable in the corresponding spaces, in spite of the fact that the supports are unbounded in each direction of $\mathbb{R}^{d}$.

\subsection{Notation}

We use the standard multi-dimensional notation in $\mathbb{R}^{d}$ and $\mathbb{N}_{0}^{d}$ as well as the standard notation concerning the known spaces of real- or complex-valued functions on $\mathbb{R}^{d}: L^{r}\left(\mathbb{R}^{d}\right)$ for $r \in[1, \infty]$ (with the norm denoted by $\|\cdot\|_{r}$ ), $L_{\text {loc }}^{1}\left(\mathbb{R}^{d}\right), \mathcal{C}\left(\mathbb{R}^{d}\right), \mathcal{C}^{\infty}\left(\mathbb{R}^{d}\right), \mathcal{E}\left(\mathbb{R}^{d}\right), \mathcal{D}\left(\mathbb{R}^{d}\right), \mathcal{S}\left(\mathbb{R}^{d}\right)$ as well as the spaces $\mathcal{D}^{\prime}\left(\mathbb{R}^{d}\right)$ of distributions and $\mathcal{S}^{\prime}\left(\mathbb{R}^{d}\right)$ of tempered distributions on $\mathbb{R}^{d}$ with the respective topologies (cf. [25], [1]).

For a given set $E \subseteq \mathbb{R}^{d}$ and a function $\phi$ on $\mathbb{R}^{d}$, we will use the following convenient notation: $E \sqsubset \mathbb{R}^{d}$ if $E$ is a compact subset of $\mathbb{R}^{d}$ and

$$
E^{\triangle}:=\left\{(x, y) \in \mathbb{R}^{2 d}: x+y \in E\right\} ; \quad \phi^{\triangle}(x, y):=\phi(x+y), \quad x, y \in \mathbb{R}^{d} .
$$

In sections 5.6 and 5.7, we will consider the space $\mathcal{D}^{\prime\left(M_{p}\right)}\left(\mathbb{R}^{d}\right)$ of Beurling ultradistributions and the space $\mathcal{S}^{\prime\left(M_{p}\right)}\left(\mathbb{R}^{d}\right)$ of Beurling tempered ultradistributions for a given sequence $\left(M_{p}\right)$ of positive numbers satisfying the following three conditions:

(M.1) $\quad M_{p}^{2} \leq M_{p-1} M_{p+1} \quad$ for $p \in \mathbb{N}$;

(M.2) $\quad M_{p} \leq A H^{p} M_{q} M_{p-q} \quad$ for $p, q \in \mathbb{N}, 0 \leq q \leq p ;$

(M.3) $\quad \sum_{p=q+1}^{\infty} M_{p-1} M_{p}^{-1} \leq A q M_{q} M_{p}^{-1} \quad$ for $q \in \mathbb{N}$,

where $A>0$ and $H>0$ are certain constants.

It will be convenient to extend the sequence $\left(M_{p}\right)$ (for $p \in \mathbb{N}_{0}$ ) to its multidimensional version $\left(M_{k}\right)$ (for $k \in \mathbb{N}_{0}^{d}$ ) in the following way:

$$
M_{k}:=M_{\kappa_{1}+\ldots+\kappa_{d}} \quad \text { for } k=\left(\kappa_{1}, \ldots, \kappa_{d}\right) \in \mathbb{N}_{0}^{d} .
$$

By the associated function for the sequence $\left(M_{p}\right)$ we will mean the function $M:[0, \infty) \rightarrow[0, \infty)$ given by $M(t):=\sup _{p \in \mathbb{N}} \log _{+}\left(t^{p} / M_{p}\right)$ for $t>0$, where $\log _{+}:=\max \{\log t, 0\}$ for $t>0$ and $M(0):=0$. 


\subsection{Compatibile sets}

We formulate certain equivalent forms of the known condition, connected with the existence of the convolution of distributions, for given closed sets in $\mathbb{R}^{d}$ :

Proposition 5.1 (see e.g. [6], p. 383). Let $A, B \subseteq \mathbb{R}^{d}$ be arbitrary closed sets. The following conditions are equivalent:

( $\Sigma) \quad(A \times B) \cap K^{\triangle} \sqsubset \mathbb{R}^{2 d} \quad$ for every $K \sqsubset \mathbb{R}^{d}$;

$\left(\Sigma^{\prime}\right) \quad A \cap(K-B) \sqsubset \mathbb{R}^{d} \quad$ for every $K \sqsubset \mathbb{R}^{d}$;

$\left(\Sigma^{\prime \prime}\right) \quad(K-A) \cap B \sqsubset \mathbb{R}^{d} \quad$ for every $K \sqsubset \mathbb{R}^{d}$.

The meaning of the conditions for $d=1$ can be seen on Fig. 5.1 (for $X=Y=$ $\mathbb{R})$.

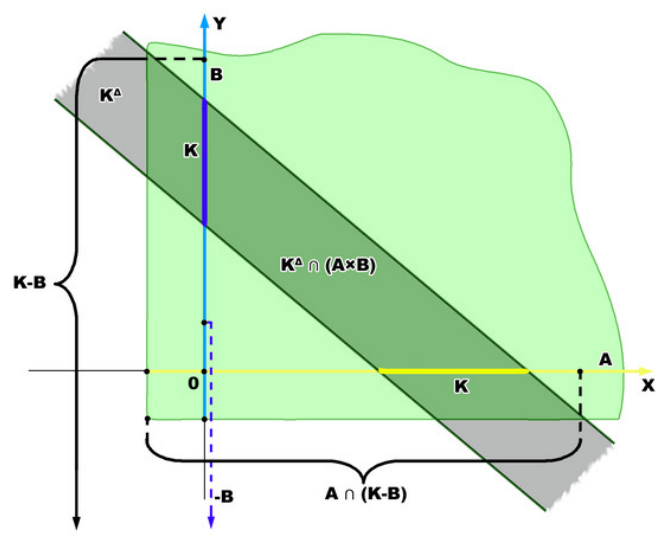

Fig. 5.1

In general, without the assumption that the sets $A$ and $B$ are closed, we have the following equivalence:

Proposition 5.2 (see [11]). Let $A, B \subseteq \mathbb{R}^{d}$ be arbitrary sets. The following conditions are equivalent:

$\left(\Sigma_{b}\right) \quad(A \times B) \cap K^{\Delta}$ is bounded in $\mathbb{R}^{2 d}$ for every bounded set $K$ in $\mathbb{R}^{d} ;$

$\left(\Sigma_{b}^{\prime}\right) \quad A \cap(K-B)$ is bounded in $\mathbb{R}^{d}$ for every bounded set $K$ in $\mathbb{R}^{d}$;

$\left(\Sigma_{b}^{\prime \prime}\right) \quad(K-A) \cap B$ is bounded in $\mathbb{R}^{d}$ for every bounded set $K$ in $\mathbb{R}^{d}$;

(M) if $x_{n} \in A$ and $y_{n} \in B$ for $n \in \mathbb{N}$, then $\left|x_{n}\right|+\left|y_{n}\right| \rightarrow \infty$ as $n \rightarrow \infty$ implies $\left|x_{n}+y_{n}\right| \rightarrow \infty$ as $n \rightarrow \infty$.

If $A, B$ are closed, then each of the above conditions is equivalent to any of conditions $(\Sigma)-\left(\Sigma^{\prime \prime}\right)$. 
Definition 5.3 (see [17], [1], [11]). Sets $A, B \subseteq \mathbb{R}^{d}$ are called compatible if any of equivalent conditions $\left(\Sigma_{b}\right)-(M)$ is satisfied.

There are two well known particular cases of compatible sets $A, B$ in $\mathbb{R}^{1}$ :

$1^{\circ}$ at least one of the sets $A, B$ is bounded (see the lower part of Fig. 5.2);

$2^{\circ}$ both sets $A, B$ are bounded from the same side: both from the left or both from the right (see the upper part of Fig. 5.2).

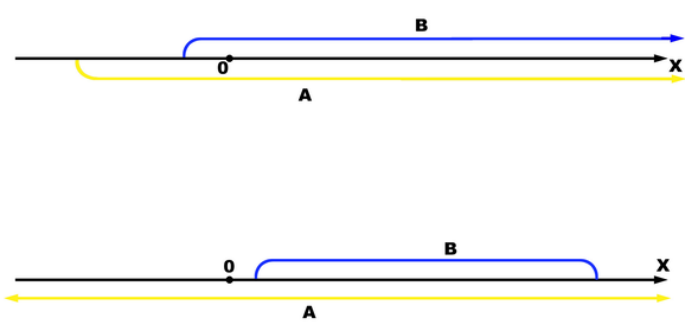

Fig. 5.2

Case $1^{\circ}$ extends clearly to $\mathbb{R}^{d}$ for $d>1$ and case $2^{\circ}$ can be described in $\mathbb{R}^{d}$ in the following form: $A, B \subset \mathbb{R}^{d}$ are (or are contained in) suitable cones with vertices at 0 such that $A$ is an open convex cone and $B \subset A^{*}$, where $A^{*}$ means the cone dual to $A$ (see [2], pp. 4-6; [1], pp. 129-130; [29], pp. 63-64). For $d=2$, case $2^{\circ}$ is illustrated on Fig. 5.3.

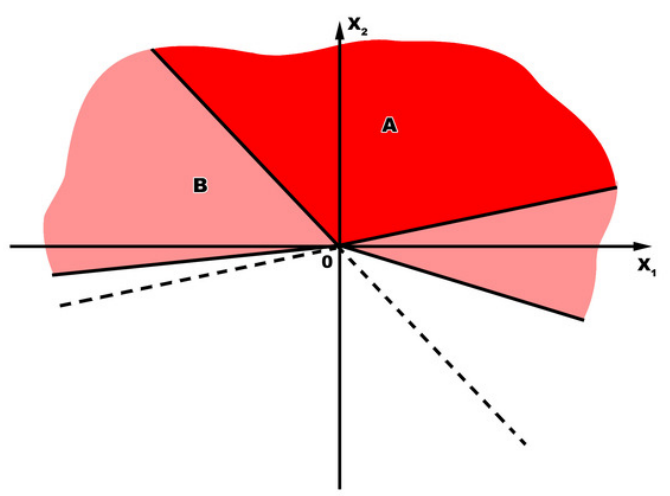

Fig. 5.3 


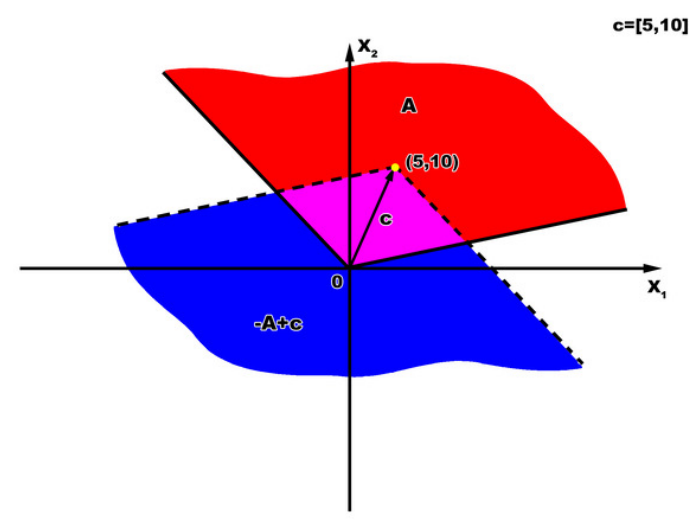

Fig. 5.4

That condition $\left(\Sigma_{b}^{\prime}\right)$ is satisfied in this case can be seen from Fig. 5.3 and 5.4 (to simplify presentation we show on Fig. 5.4 the set $A \cap(c-B)$ only for $B:=A$, with $A$ as on Fig. 5.3, and for a specific $c \in \mathbb{R}^{2}$, but a general case is easily seen).

There exist, however, another case of compatible sets in $\mathbb{R}^{1}$, not so well known as $1^{\circ}$ and $2^{\circ}$ :

$3^{\circ}$ both sets $A, B$ in $\mathbb{R}^{1}$ are unbounded from both sides: unbounded both from the left and from the right.

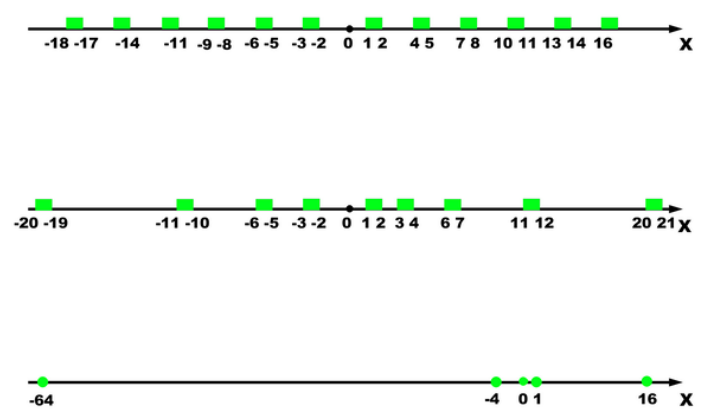

Fig. 5.5

The set presented in each of the three parts of Fig. 5.5, let us denote it by the common symbol $A$, is a union of countably many intervals of length 1 situated in three different ways on $\mathbb{R}^{1}$. The set $A$ in the two lower parts of Fig. 5.5 is compatible with itself and the set $A$ in the upper part of Fig. 5.5 is not compatible with itself (for details see [7], [8] and [15]). 


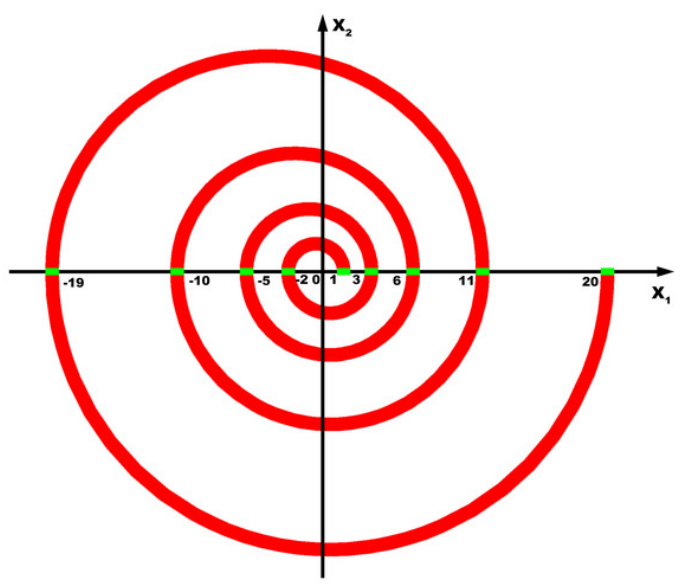

Fig. 5.6

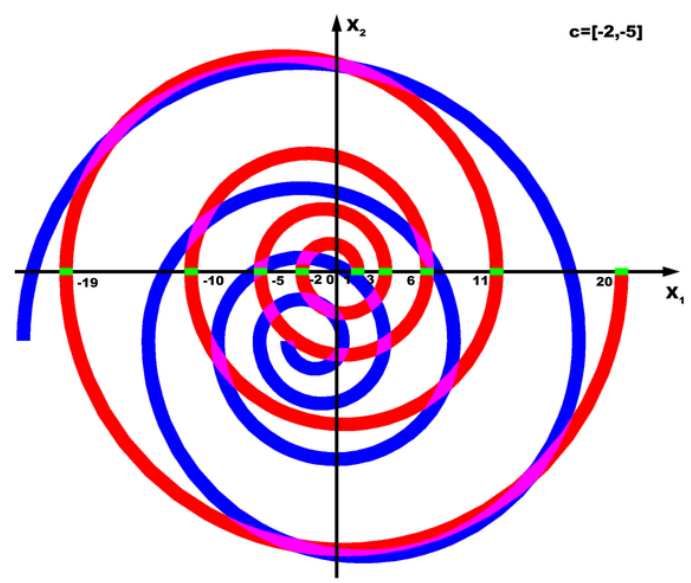

Fig. 5.7

Case $3^{\circ}$ can be extended to $\mathbb{R}^{d}$ in various manners. An interesting extension are sets which can be described as infinite spirals or helices with a suitable way of developing their coils in $\mathbb{R}^{d}$. Notice that compatible sets which are unbounded in each direction of $\mathbb{R}^{d}$ can be obtained in this way (see [15]).

On Fig. 5.6 and 5.8 particular examples of such a spiral set $A$ in $\mathbb{R}^{2}$ which is compatible with itself are shown. On Fig. 5.7 and 5.9 (analogously to Fig. 5.4) we show only the set $A \cap(c-A)$ for specific vectors $c \in \mathbb{R}^{2}$. 


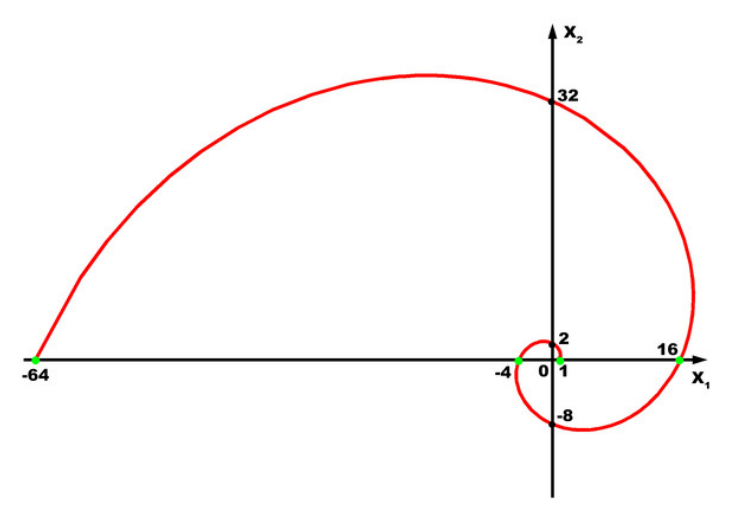

Fig. 5.8

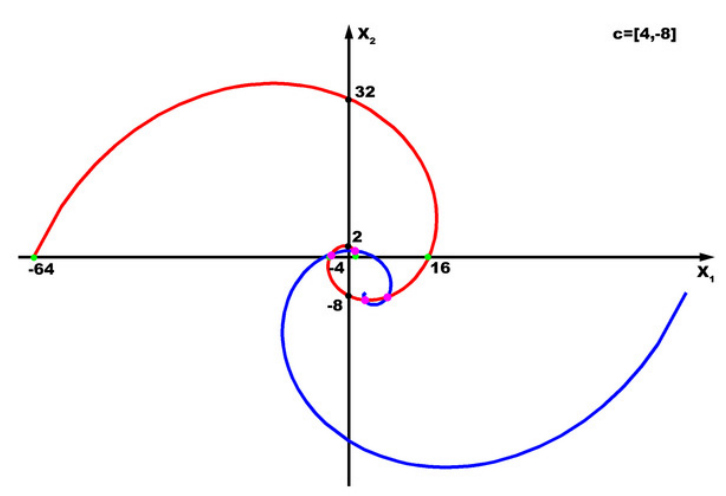

Fig. 5.9

Compatibility of supports of two tempered distributions, elements of the subspace $\mathcal{S}^{\prime}\left(\mathbb{R}^{d}\right)$ of the space $\mathcal{D}^{\prime}\left(\mathbb{R}^{d}\right)$, does not guarantee that their convolution in $\mathcal{D}^{\prime}$ is again a tempered distribution (see section 5.5). Similarly, compatibility of supports of two tempered ultradistributions, elements of the subspace $\mathcal{S}^{\prime\left(M_{p}\right)}\left(\mathbb{R}^{d}\right)$ of the space $\mathcal{D}^{\prime\left(M_{p}\right)}\left(\mathbb{R}^{d}\right)$, does not guarantee that their convolution in $\mathcal{D}^{\prime\left(M_{p}\right)}$ is again a tempered ultradistribution (see sections 5.6 and 5.7). This is a consequence of Theorem 5.9 formulated in the next section.

Therefore the notion of compatibility requires suitable modifications in the spaces $\mathcal{S}^{\prime}\left(\mathbb{R}^{d}\right)$ of tempered distributions and $\mathcal{S}^{\prime\left(M_{p}\right)}\left(\mathbb{R}^{d}\right)$ of tempered ultradistributions.

In [7], [8], the following modifications of compatibility, corresponding to the mentioned spaces were introduced. 
Definition 5.4 (see [7], [8], [10]). Two sets $A, B \subseteq \mathbb{R}^{d}$ are polynomially compatible if there is a positive polynomial $p$ on $[0, \infty)$ such that the following implication holds:

$$
x \in A, y \in B \Rightarrow|x|+|y| \leq p(|x+y|)
$$

or, equivalently, if there are $C>0$ and $k \in \mathbb{N}_{0}$ such that

$$
x \in A, y \in B \Rightarrow|x|+|y| \leq C(1+|x+y|)^{k} .
$$

In [14], a certain modification of compatibility condition, corresponding to the space $\mathcal{S}^{\prime\left(M_{p}\right)}\left(\mathbb{R}^{d}\right)$, was given via the associated function $M$ for the sequence $\left(M_{p}\right)$. We present it here in a slightly relaxed form:

Definition 5.5 (cf. [14]). Two sets $A, B \subseteq \mathbb{R}^{d}$ are $M$-compatible, if there is a constant $a>0$ such that

$$
M(|x|)+M(|y|) \leq M(a|x+y|)+a
$$

for all $x \in A$ and $y \in B$.

\subsection{Existence of convolution in $L_{\text {loc }}^{1}$}

Definition 5.6. Let $F$ and $G$ be Lebesgue measurable functions on $\mathbb{R}^{d}$. For a given $x \in \mathbb{R}^{d}$ we define

$$
(F * G)(x):=\int_{\mathbb{R}^{d}} F(x-t) G(t) d t,
$$

saying that $(F * G)(x)$ exists, whenever the function under the integral sign in (5.1) is Lebesgue integrable as a function of $t$ for the fixed $x$. If $(F * G)(x)$ exists for all (almost all) $x \in \mathbb{R}^{d}$, we say that the convolution $F * G$ exists everywhere (almost everywhere) on $\mathbb{R}^{d}$.

Definition 5.7 (see [15]). Let $F$ and $G$ be functions in $L_{\text {loc }}^{1}\left(\mathbb{R}^{d}\right)$. We say that the convolution $F * G$ exists in $L_{\text {loc }}^{1}$ if $F * G$ exists almost everywhere on $\mathbb{R}^{d}$ and $|F| *|G| \in L_{\mathrm{loc}}^{1}\left(\mathbb{R}^{d}\right)$.

Let us recall the known particular case (Young's theorem) of the existence of the convolution in $L_{\text {loc }}^{1}$ of functions from suitable subspaces $L^{p}\left(\mathbb{R}^{d}\right)$ and $L^{q}\left(\mathbb{R}^{d}\right)$ of the space $L_{\text {loc }}^{1}\left(\mathbb{R}^{d}\right)$ : 
If $F \in L^{p}\left(\mathbb{R}^{d}\right)$ and $G \in L^{q}\left(\mathbb{R}^{d}\right)$ for $p, q \in[1, \infty)$ such that $1 / p+1 / q \geq 1$, then the convolution $F * G$ exists almost everywhere in $\mathbb{R}^{d}$ and $F * G \in L^{r}\left(\mathbb{R}^{d}\right)$, where $r=(1 / p+1 / q-1)^{-1}$. In particular, if $F, G \in L^{1}\left(\mathbb{R}^{d}\right)$, then the convolution $F * G$ exists almost everywhere in $\mathbb{R}^{d}$ and $F * G \in L^{1}\left(\mathbb{R}^{d}\right)$.

The convolution in $L_{\text {loc }}^{1}$ of two locally integrable functions may not exist, but it exists if the supports of the functions are compatible (see section 5.3):

Theorem 5.8 (see e.g. [1], p. 124). If $F, G \in L_{\mathrm{loc}}^{1}\left(\mathbb{R}^{d}\right)$ and the supports of the functions $F$ and $G$ are (contained in) compatible sets, then the convolution $F * G$ exists in $L_{\text {loc }}^{1}$.

However the existence of the convolution of functions in $L_{\mathrm{loc}}^{1}$ (similarly, the existence of the convolution of distributions or of ultradistributions in the respective spaces, see sections 5.5 and 5.6) does not guarantee any restriction of growth of the convolution. For instance, the convolution of two measurable slowly increasing functions may exist in $L_{\mathrm{loc}}^{1}$ but their convolution may be a function of arbitrarily fast increase. As a matter of fact, the following much stronger result was proved in [7] (see also [8] and [15]):

Theorem 5.9 (see [7], [8], [15]). Let $F \in \mathcal{C}\left(\mathbb{R}^{d}\right)$ be an arbitrary continuous nonnegative function (of an arbitrary increase). Then there exists a nonnegative smooth function $\phi \in \mathcal{C}^{\infty}\left(\mathbb{R}^{d}\right)$ such that its support is compatible with itself and the convolution $\varphi * \varphi$ satisfies the inequality $(\phi * \phi)(x)>F(x)$ for each $x \in \mathbb{R}^{d}$. Moreover, $\lim _{|x| \rightarrow \infty} \phi(x)=0$.

Due to its strong formulation the above theorem can serve as a universal counter-example in considerations concerning the existence and the growth of the convolution of functions, distributions and ultradistributions.

\subsection{Existence of convolution in $\mathcal{D}^{\prime}$ and in $\mathcal{S}^{\prime}$}

There are several general definitions of the convolution of distributions in $\mathcal{D}^{\prime}$ given consecutively by C. Chevalley [3], L. Schwartz [26], R. Shiraishi [27], V. S. Vladimirov [28], [29], P. Dierolf - J. Voigt [4], A. Kamiński [9] and S. Mincheva-Kamińska [19], [20] (see also [32], [5], [30], [31], [21], [18] and [15]). These general definitions allow one to define the convolution $f * g$ in $\mathcal{D}^{\prime}$ for arbitrary distributions $f, g \in \mathcal{D}^{\prime}\left(\mathbb{R}^{d}\right)$ and to determine, for each pair $(f, g)$ of distributions, whether the convolution $f * g$ exists in $\mathcal{D}^{\prime}$ (then $f * g \in \mathcal{D}^{\prime}\left(\mathbb{R}^{d}\right)$ ) or not. Most of the mentioned definitions are equivalent (for details see e.g. 
[9]). We will recall only one of them, the sequential definition of Vladimirov [28], [29], based on the notion of strong approximate unit.

Definition 5.10 (see [28], [29], [4], [9]). A sequence $\left(\eta_{n}\right)$ of elements of $\mathcal{D}\left(\mathbb{R}^{d}\right)$ is said to be a strong approximate unit on $\mathbb{R}^{d}$ if for every $K \sqsubset \mathbb{R}^{d}$ there exists an $n_{0} \in \mathbb{N}$ such that $\eta_{n}(x)=1$ for $x \in K$ and $n \geq n_{0}$ (hence $\eta_{n} \rightarrow 1$ in $\mathcal{E}\left(\mathbb{R}^{d}\right)$ ) and, in addition,

$$
\sup _{n \in \mathbb{N}}\left\|\eta_{n}^{(k)}\right\|_{\infty}<\infty \quad \text { for every } k \in \mathbb{N}_{0}^{d} .
$$

We denote the set of all strong approximate units on $\mathbb{R}^{d}$ by $\overline{\mathbb{U}}\left(\mathbb{R}^{d}\right)$.

Definition 5.11 (see [28], [29], [4], [9]). For given $f, g \in \mathcal{D}^{\prime}\left(\mathbb{R}^{d}\right)$ the convolution $f * g$ in $\mathcal{D}^{\prime}$ is defined by

$$
\langle f * g, \varphi\rangle:=\lim _{n \rightarrow \infty}\left\langle f \otimes g, \eta_{n} \varphi^{\triangle}\right\rangle, \quad \varphi \in \mathcal{D}\left(\mathbb{R}^{d}\right),
$$

whenever the above limit exists for every strong approximate unit $\left(\eta_{n}\right) \in$ $\overline{\mathbb{U}}\left(\mathbb{R}^{2 d}\right)$ and $\varphi \in \mathcal{D}\left(\mathbb{R}^{d}\right)$. We say then that the convolution $f * g$ exists in $\mathcal{D}^{\prime}$.

Recall that the space $\mathcal{S}^{\prime}\left(\mathbb{R}^{d}\right)$ of tempered distributions is a subspace of the space $\mathcal{D}^{\prime}\left(\mathbb{R}^{d}\right)$ of distributions. Analogously to and independently of the above Definition 5.11, one may define the convolution of tempered distributions in $\mathcal{S}^{\prime}$ in various ways (see e.g. [27], [4], [9] and other references given earlier). We present below only one of several equivalent definitions of the convolution in $\mathcal{S}^{\prime}$, namely the respective counterpart of the above sequential definition of Vladimirov (cf. [28], [29]):

Definition 5.12 (see [4], [9]). For given $f, g \in \mathcal{S}^{\prime}\left(\mathbb{R}^{d}\right)$ we define the convolution $f * g$ in $\mathcal{S}^{\prime}$ by

$$
\langle f * g, \psi\rangle:=\lim _{n \rightarrow \infty}\left\langle f \otimes g, \eta_{n} \psi^{\triangle}\right\rangle, \quad \psi \in \mathcal{S}\left(\mathbb{R}^{d}\right),
$$

whenever the above limit exists for every strong approximate unit $\left(\eta_{n}\right) \in$ $\overline{\mathbb{U}}\left(\mathbb{R}^{2 d}\right)$ and $\psi \in \mathcal{S}\left(\mathbb{R}^{d}\right)$. We say then that the convolution $f * g$ exists in $\mathcal{S}^{\prime}$.

For existence of the convolution in $\mathcal{D}^{\prime}$ of two distributions $f, g \in \mathcal{D}^{\prime}\left(\mathbb{R}^{d}\right)$ the condition, introduced in section 5.4, of compatibility of their supports is sufficient. Namely

Theorem 5.13 (see [1]; cf. [6], [28], [29]). Let $f, g \in \mathcal{D}^{\prime}\left(\mathbb{R}^{d}\right)$ be distributions. If the supports of $f$ and $g$ are (contained in) compatible sets in $\mathbb{R}^{d}$, then $f * g$ exists in $\mathcal{D}^{\prime}$ and $f * g \in \mathcal{D}^{\prime}\left(\mathbb{R}^{d}\right)$. 
In [27], R. Shiraishi posed the problem whether the assumption that the convolution $f * g$ of two tempered distributions $f, g \in \mathcal{S}^{\prime}\left(\mathbb{R}^{d}\right)\left(\subset \mathcal{D}^{\prime}\left(\mathbb{R}^{d}\right)\right)$ exists in $\mathcal{D}^{\prime}$ implies that the convolution $f * g$ exists in $\mathcal{S}^{\prime}$, in particular, whether the existence in $\mathcal{D}^{\prime}$ of the convolution $f * g$ of tempered distributions $f, g \in \mathcal{S}^{\prime}\left(\mathbb{R}^{d}\right)$ implies that the convolution $f * g$ not only belongs to $\mathcal{D}^{\prime}\left(\mathbb{R}^{d}\right)$, but is even a member of $\mathcal{S}^{\prime}\left(\mathbb{R}^{d}\right)$.

The negative answer to the problem of Shiraishi follows directly from Theorem 5.9 recalled in section 5.4, proved in [7], [8] (see also [15]), and from the paper [4]. In [4], an example of two tempered measures $f, g$ is given, concentrated on a countable set in $\mathbb{R}^{1}$, such that the convolution $f * g$ exists in $\mathcal{D}^{\prime}$, but $f * g \notin \mathcal{S}^{\prime}\left(\mathbb{R}^{1}\right)$. Theorem 5.9 is much stronger than the result from [4] and stands for a counter-example concerning the convolution in various spaces of functions and generalized functions.

In particular, it follows from Theorem 5.9 that the counterpart of Theorem 5.13 for tempered distributions is not true under the assumption of compatibility of their supports. However, if one replaces this assumption by polynomial compatibility of supports of given tempered distributions, the result concerning their convolution in $\mathcal{S}^{\prime}$ is analogous:

Theorem 5.14 (see [7], [8], [10]). Let $f, g \in \mathcal{S}^{\prime}\left(\mathbb{R}^{d}\right)$ be tempered distributions. If the supports of $f$ and $g$ are (contained in) polynomially compatible sets in $\mathbb{R}^{d}$, then the convolution $f * g$ exists in $\mathcal{S}^{\prime}$ and $f * g \in \mathcal{S}^{\prime}\left(\mathbb{R}^{d}\right)$.

\subsection{Definition of spaces $\mathcal{D}^{\prime\left(M_{p}\right)}$ and $\mathcal{S}^{\prime\left(M_{p}\right)}$}

We recall the definition of Beurling spaces of ultradifferentiable functions for a fixed numerical sequence $\left(M_{p}\right)$ satisfying conditions $(M .1)-(M .3)$, formulated in section 5.2, which will be assumed to the end of this and the next section.

We start with defining, for given $h>0$ and regular compact subset $K$ of $\mathbb{R}^{d}$ (see [16]), the spaces $\mathcal{E}_{K, h}^{\left(M_{p}\right)}\left(\mathbb{R}^{d}\right)$ and $\mathcal{D}_{K, h}^{\left(M_{p}\right)}\left(\mathbb{R}^{d}\right)$. The space $\mathcal{E}_{K, h}^{\left(M_{p}\right)}\left(\mathbb{R}^{d}\right)$ is defined to consist of all functions $\varphi$ from $\mathcal{E}\left(\mathbb{R}^{d}\right)$ such that

$$
\|\varphi\|_{K, h}:=\sup _{k \in \mathbb{N}_{0}^{d} d \in K} \frac{\left|\varphi^{(k)}(x)\right|}{h^{k} M_{k}}<\infty
$$

and $\mathcal{D}_{K, h}^{\left(M_{p}\right)}\left(\mathbb{R}^{d}\right)$ denotes the space of all $\varphi \in \mathcal{E}\left(\mathbb{R}^{d}\right)$ with the support contained in $K$, satisfying inequality (5.2). Then we define the following basic spaces of functions: 


$$
\begin{aligned}
& \mathcal{E}^{\left(M_{p}\right)}\left(\mathbb{R}^{d}\right):=\underset{K \subset \subset \mathbb{R}^{d}}{\operatorname{proj} \operatorname{pim}} \underset{h \rightarrow 0}{\operatorname{proj} \lim } \mathcal{E}_{K, h}^{\left(M_{p}\right)}\left(\mathbb{R}^{d}\right) ;
\end{aligned}
$$

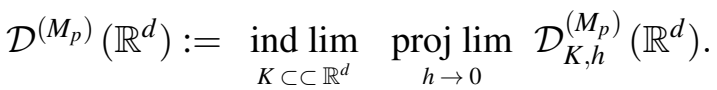

The symbol $K \subset \subset \mathbb{R}^{d}$ above means that the sets $K$ are regular compact (see [16]) and grow up to $\mathbb{R}^{d}$.

Moreover we define first $\mathcal{D}_{L^{s}, h}^{\left(M_{p}\right)}\left(\mathbb{R}^{d}\right)$, for given $h>0$ and $s \in[1, \infty]$, as the space of all functions $\varphi$ from $C^{\infty}\left(\mathbb{R}^{d}\right)$ satisfying the inequality:

$$
\|\varphi\|_{s, h}:=\sup \left\{\frac{\left\|\varphi^{(k)}\right\|_{s}}{h^{k} M_{k}}: k \in \mathbf{N}_{0}^{d}\right\}<\infty,
$$

and then we define

$$
\mathcal{D}_{L^{s}}^{\left(M_{p}\right)}\left(\mathbb{R}^{d}\right):=\underset{h \rightarrow 0}{\operatorname{proj} \lim } \mathcal{D}_{L^{s}, h}^{\left(M_{p}\right)}\left(\mathbb{R}^{d}\right) .
$$

By $\dot{\mathcal{B}}^{\left(M_{p}\right)}\left(\mathbb{R}^{d}\right)$, we denote the completion of $\mathcal{D}^{\left(M_{p}\right)}\left(\mathbb{R}^{d}\right)$ in $\mathcal{D}_{L^{\infty}}^{\left(M_{p}\right)}\left(\mathbb{R}^{d}\right)$. For more details concerning all the above spaces we refer to [16], [23], [24], [12], [13] and [2].

In addition, for a fixed $m>0$, we denote by $\mathcal{S}_{2}^{\left(M_{p}\right), m}\left(\mathbb{R}^{d}\right)$ the space of all smooth functions $\varphi$ such that

$$
\sigma_{m, 2}(\varphi):=\left(\sum_{\alpha, \beta \in \mathbb{N}_{0}^{d}} \frac{m^{2(\alpha+\beta)}}{M_{\alpha}^{2} M_{\beta}^{2}} \int_{\mathbb{R}^{d}}\left|\langle x\rangle^{\beta} \varphi^{(\alpha)}(x)\right|^{2} d x\right)^{1 / 2}<\infty,
$$

where $\langle x\rangle:=\left(1+|x|^{2}\right)^{1 / 2}$ for $x \in \mathbb{R}^{d}$, equipped with the topology induced by the above norm $\sigma_{m, 2}$, Then we define

$$
\mathcal{S}^{\left(M_{p}\right)}\left(\mathbb{R}^{d}\right):=\operatorname{proj} \lim \mathcal{S}_{2}^{\left(M_{p}\right), m}\left(\mathbb{R}^{d}\right) .
$$

For more details concerning the above spaces we refer to [22], [14] and [2]).

Remark 5.15. Notice that the basic spaces $\mathcal{E}^{\left(M_{p}\right)}\left(\mathbb{R}^{d}\right), \mathcal{D}^{\left(M_{p}\right)}\left(\mathbb{R}^{d}\right), \mathcal{D}_{L^{s}}^{\left(M_{p}\right)}\left(\mathbb{R}^{d}\right)$ for $s \in[1, \infty], \dot{\mathcal{B}}^{\left(M_{p}\right)}\left(\mathbb{R}^{d}\right)$ and $\mathcal{S}^{\left(M_{p}\right)}\left(\mathbb{R}^{d}\right)$ contain sufficiently many functions (in case of the space $\mathcal{D}^{\left(M_{p}\right)}\left(\mathbb{R}^{d}\right)$, this is a consequence of the Denjoy-Carleman theorem). In particular, there exists a function $\eta \in \mathcal{D}^{\left(M_{p}\right)}\left(\mathbb{R}^{d}\right)$ such that $\eta=1$ in some neighbourhood of 0 . 
The strong dual of $\mathcal{D}^{\left(M_{p}\right)}\left(\mathbb{R}^{d}\right)$, denoted by $\mathcal{D}^{\prime\left(M_{p}\right)}\left(\mathbb{R}^{d}\right)$, is called the space of Beurling ultradistributions.

Notice that $\mathcal{D}^{\left(M_{p}\right)}\left(\mathbb{R}^{d}\right)$ is dense in $\mathcal{D}_{L^{s}}^{\left(M_{p}\right)}\left(\mathbb{R}^{d}\right)$ for $s \in[1, \infty)$ as well as in $\dot{\mathcal{B}}^{\left(M_{p}\right)}\left(\mathbb{R}^{d}\right)$; moreover, the respective inclusion mappings are continuous. Hence the strong duals of $\mathcal{D}_{L^{s}}^{\left(M_{p}\right)}\left(\mathbb{R}^{d}\right)(s \in[1, \infty))$ and $\dot{\mathcal{B}}^{\left(M_{p}\right)}\left(\mathbb{R}^{d}\right)$ are subspaces of the space $\mathcal{D}^{\prime\left(M_{p}\right)}\left(\mathbb{R}^{d}\right)$ of all Beurling ultradistributions. We denote them traditionally by $\mathcal{D}_{L^{t}}^{\left(M_{p}\right)}\left(\mathbb{R}^{d}\right)$, where $t:=s /(s-1) \in(1, \infty]$, and $\mathcal{D}_{L^{1}}^{\left(M_{p}\right)}\left(\mathbb{R}^{d}\right)$, respectively (see [16], [2]).

The space $\mathcal{S}^{\left(M_{p}\right)}\left(\mathbb{R}^{d}\right)$ of all Beurling tempered ultradistributions is meant as the strong dual of the space $\mathcal{S}^{\left(M_{p}\right)}\left(\mathbb{R}^{d}\right)$ defined above (see [22], [14], [2]). Since $\mathcal{D}^{\left(M_{p}\right)}\left(\mathbb{R}^{d}\right)$ is dense in $\mathcal{S}^{\left(M_{p}\right)}\left(\mathbb{R}^{d}\right)$ and the inclusion mapping is continuous, so $\mathcal{S}^{\prime\left(M_{p}\right)}\left(\mathbb{R}^{d}\right)$ can be embedded into the space $\mathcal{D}^{\prime\left(M_{p}\right)}\left(\mathbb{R}^{d}\right)$. For other properties of the space $\mathcal{S}^{\prime\left(M_{p}\right)}$ we refer to [22], [14] and [2].

For given ultradistributions $f, g \in \mathcal{D}^{\prime\left(M_{p}\right)}\left(\mathbb{R}^{d}\right)$ by their tensor product $f \otimes g$ we mean an ultradistribution in $\mathcal{D}^{\prime\left(M_{p}\right)}\left(\mathbb{R}^{2 d}\right)$ defined in a standard way.

\subsection{Existence of convolution in $\mathcal{D}^{\prime\left(M_{p}\right)}$ and in $\mathcal{S}^{\prime\left(M_{p}\right)}$}

There are various general definitions of the convolutions in $\mathcal{D}^{\prime\left(M_{p}\right)}$ of Beurling ultradistributions (see [12]) and in $\mathcal{S}^{\prime\left(M_{p}\right)}$ of Beurling tempered ultradistributions (see [14]). They are counterparts of the known general definitions of the convolutions in $\mathcal{D}^{\prime}$ and in $\mathcal{S}^{\prime}$ (see section 5).

That the mentioned definitions of the convolution in $\mathcal{D}^{\prime\left(M_{p}\right)}$ of Beurling ultradistributions are equivalent and that the corresponding definitions of the convolution in $\mathcal{S}^{\left(M_{p}\right)}$ of Beurling tempered ultradistributions are equivalent was proved in [12] and [14], respectively (see also [2]).

We will recall here only these definitions of the convolution in $\mathcal{D}^{\prime\left(M_{p}\right)}$ and in $\mathcal{S}^{\prime\left(M_{p}\right)}$ which correspond to Vladimirov's definition of the convolution in $\mathcal{D}^{\prime}$ and in $\mathcal{S}^{\prime}$, respectively. The definitions are based on the notions of strong $\mathcal{D}^{\left(M_{p}\right)}$-approximate unit and strong $\mathcal{S}^{\left(M_{p}\right)}$-approximate unit.

Definition 5.16 (see [12], [13]). A sequence $\left(\eta_{n}\right)$ of elements of $\mathcal{D}^{\left(M_{p}\right)}\left(\mathbb{R}^{d}\right)$ is said to be a strong $\mathcal{D}^{\left(M_{p}\right)}$-approximate unit on $\mathbb{R}^{d}$ if for every $K \sqsubset \mathbb{R}^{d}$ there exists an $n_{0} \in \mathbb{N}$ such that $\eta_{n}(x)=1$ for $x \in K$ and $n \geq n_{0}$ (hence $\eta_{n} \rightarrow 1$ in $\left.\mathcal{E}^{\left(M_{p}\right)}\left(\mathbb{R}^{d}\right)\right)$ and, in addition, if there exists a positive constant $h$ such that

$$
\sup _{n \in \mathbb{N}} \sup _{k \in \mathbb{N}_{0}^{d}}\left(\frac{h^{k}}{M_{k}}\left\|\eta_{n}^{(k)}\right\|_{\infty}\right)<\infty .
$$


We denote the set of all strong $\mathcal{D}^{\left(M_{p}\right)}$-approximate units on $\mathbb{R}^{d}$ by $\overline{\mathbb{U}}^{\left(M_{p}\right)}\left(\mathbb{R}^{d}\right)$.

Definition 5.17 (see [13], [14]). If in the above definition the assumption $\eta_{n} \in$ $\mathcal{D}^{\left(M_{p}\right)}\left(\mathbb{R}^{d}\right)$ for $n \in \mathbb{N}$ is replaced by $\eta_{n} \in \mathcal{S}^{\left(M_{p}\right)}\left(\mathbb{R}^{d}\right)$ for $n \in \mathbb{N}$ and the remaining assumptions are preserved, then the sequence $\left(\eta_{n}\right)$ is called a strong $\mathcal{S}^{\left(M_{p}\right)}$ approximate unit. We denote the set of all strong $\mathcal{S}^{\left(M_{p}\right)}$-approximate units on $\mathbb{R}^{d}$ by $\overline{\mathbb{U}}_{s}^{\left(M_{p}\right)}\left(\mathbb{R}^{d}\right)$.

Vladimirov's version of the definition of the convolution in $\mathcal{D}^{\prime\left(M_{p}\right)}$ of Berling ultradistributions has the following form:

Definition 5.18 (see [12], [13]). For given Beurling ultradistributions $f, g \in$ $\mathcal{D}^{\prime\left(M_{p}\right)}\left(\mathbb{R}^{d}\right)$ the convolution $f * g$ in $\mathcal{D}^{\prime\left(M_{p}\right)}$ is defined by

$$
\langle f * g, \varphi\rangle:=\lim _{n \rightarrow \infty}\left\langle f \otimes g, \eta_{n} \varphi^{\triangle}\right\rangle, \quad \varphi \in \mathcal{D}^{\left(M_{p}\right)}\left(\mathbb{R}^{d}\right),
$$

whenever the above limit exists for every strong approximate unit $\left(\eta_{n}\right) \in$ $\overline{\mathbb{U}}^{\left(M_{p}\right)}\left(\mathbb{R}^{2 d}\right)$ and $\varphi \in \mathcal{D}^{\left(M_{p}\right)}\left(\mathbb{R}^{d}\right)$. We say then that the convolution $f * g$ exists in $\mathcal{D}^{\prime}\left(M_{p}\right)$.

Analogously, the convolution in $\mathcal{S}^{\prime\left(M_{p}\right)}$ of Beurling tempered ultradistributions can be defined as follows:

Definition 5.19 (see [13], [14]). For given two Beurling tempered ultradistributions $f, g \in \mathcal{S}^{\prime\left(M_{p}\right)}\left(\mathbb{R}^{d}\right)$ we define the convolution $f * g$ in $\mathcal{S}^{\prime\left(M_{p}\right)}$ by

$$
\langle f * g, \psi\rangle:=\lim _{n \rightarrow \infty}\left\langle f \otimes g, \eta_{n} \psi^{\triangle}\right\rangle, \quad \psi \in \mathcal{S}^{\left(M_{p}\right)}\left(\mathbb{R}^{d}\right),
$$

whenever the above limit exists for every strong approximate unit $\left(\eta_{n}\right) \in$ $\overline{\mathbb{U}}_{s}^{\left(M_{p}\right)}\left(\mathbb{R}^{2 d}\right)$ and $\psi \in \mathcal{S}^{\left(M_{p}\right)}\left(\mathbb{R}^{d}\right)$. We say then that the convolution $f * g$ exists in $\mathcal{S}^{\prime}\left(M_{p}\right)$.

The following analogue of Theorem 5.13 is true for the convolution in $\mathcal{D}^{\prime\left(M_{p}\right)}$ :

Theorem 5.20 (see [13]). Let $f, g \in \mathcal{D}^{\prime\left(M_{p}\right)}\left(\mathbb{R}^{d}\right)$ be Beurling ultradistributions. If the supports of $f$ and $g$ are (contained in) compatible sets in $\mathbb{R}^{d}$, then the convolution $f * g$ exists in $\mathcal{D}^{\prime\left(M_{p}\right)}$ and $f * g \in \mathcal{D}^{\prime\left(M_{p}\right)}\left(\mathbb{R}^{d}\right)$.

There is also a counterpart of Theorem 5.14 for the convolution in $\mathcal{S}^{\prime\left(M_{p}\right)}$ of Beurling tempered ultradistributions. Namely, we have 
Theorem 5.21 (see [13]). Let $f, g \in \mathcal{S}^{\prime\left(M_{p}\right)}\left(\mathbb{R}^{d}\right)$ be Beurling tempered ultradistributions. If the supports of $f$ and $g$ are (contained in) $M$-compatible sets in $\mathbb{R}^{d}$, then the convolution $f * g$ exists in $\mathcal{S}^{\left(M_{p}\right)}$ and $f * g \in \mathcal{S}^{\prime\left(M_{p}\right)}\left(\mathbb{R}^{d}\right)$.

Acknowledgements. We would like to express our gratitude to Mr. Klaudiusz Majchrowski for preparing the figures illustrating certain topics discussed in the article.

\section{References}

[1] P. Antosik, J. Mikusiński, R. Sikorski, Theory of Distributions. The Sequential Approach, Elsevier-PWN, Amsterdam-Warszawa, 1973.

[2] R. D. Carmichael, A. Kamiński, S. Pilipović, Boundary Values and Convolution in Ultradistribution Spaces, World Scientific, New Jersey, 2007.

[3] C. Chevalley, Theory of Distributions, Lectures at Columbia University, 1950-1951.

[4] P. Dierolf, J. Voigt, Convolution and $\mathcal{S}^{\prime}$-convolution of distributions, Collect. Math. 29 (1978), 185-196.

[5] Y. Hirata, On convolutions in the theory of distributions, J. Sci. Hiroshima Univ. Ser. A 22 (1958), 89-98.

[6] J. Horvath, Topological Vector Spaces and Distributions, Vol. I, Addison-Wesley, Reading-London, 1966.

[7] A. Kamiński, Integration and irregular operations, Ph.D. Thesis, Institute of Mathematics, Polish Academy of Sciences, Warsaw, 1975; Operacje regularne i nieregularne na dystrybucjach, Institute of Mathematics, Polish Academy of Sciences, Preprint 11, Ser. B, 1981.

[8] A. Kamiński, On convolutions, products and Fourier transforms of distributions, Bull. Acad. Polon. Sci. Sér. Sci. Math. Astronom. Phys. 25 (1977), 369-374.

[9] A. Kamiński, Convolution, product and Fourier transform of distributions, Studia Math. 74 (1982), 83-86.

[10] A. Kamiński, On the Rényi theory of conditional probabilities, Studia Math. 79 (1984), 151-191.

[11] A. Kamiński, Remarks on the convolution of distributions with compatible supports, Zeszyty Nauk. Politechn. Ślassk. Mat. Fiz. 64 (1990), 107-122.

[12] A. Kamiński, D. Kovačević, S. Pilipović, The equivalence of various definitions of the convolution of ultradistributions, Trudy Mat. Inst. Steklov 203 (1994), 307-322.

[13] A. Kamiński, D. Perisić, S. Pilipović, Existence theorems for convolution of ultradistributions, Dissertationes Math. 340 (1995), 79-91.

[14] A. Kamiński, D. Perišić, S. Pilipović, On the convolution of tempered ultradistributions of Beurling type, Integral Transforms Spec. Func. 15 (2003), 323-329.

[15] A. Kamiński, S. Mincheva-Kamińska, On the convolution of functions and generalized functions, submitted.

[16] H. Komatsu, Ultradistributions I-III, J. Fac. Sci. Univ. Tokyo Sect. IA Mat. 20 (1973), 25-105; 24 (1977), 607-628; 29 (1982), 653-718.

[17] J. Mikusiński, Sequential theory of convolution of distributions, Studia Math. 29 (1968), 151-160. 
[18] S. Mincheva-Kamińska, Sequential approach to integrable distributions, Novi Sad J. Math. 41 (2011), 123-131.

[19] S. Mincheva-Kamińska, Equivalence of sequential definitions of the convolution of distributions, Rend. Sem. Mat. Univ. Politec. Torino 69 (2011), 367-376.

[20] S. Mincheva-Kamińska, Equivalent conditions for integrability and convolvability of distributions and tempered distributions, submitted.

[21] N. Ortner, On convolvability conditions for distributions, Monatsh. Math. 160 (2010), 313-335.

[22] S. Pilipović, Tempered ultradistributions, Boll. Un. Mat. Ital. (7) 2-B (1988), 235-251.

[23] S. Pilipović, On the convolution in the space of Beurling ultradistributions, Comm. Math. Univ. St. Paul. 40 (1991), 15-27.

[24] S. Pilipović, Characterizations of bounded sets in the space of ultradistributions, Proc. Amer. Math. Soc. 120 (1994), 1191-1206.

[25] L. Schwartz, Théorie des distributions, Vol. 1-2, Hermann, Paris, 1950-51; nouvelle édition 1966.

[26] L. Schwartz, Définitions integrale de la convolution de deux distributions, in: Séminaire Schwartz, Année 1953-54. Expose n 22, Secr. math. Fac. Sci., Paris, 1954.

[27] R. Shiraishi, On the definition of convolution for distributions, J. Sci. Hiroshima Univ. Ser. A 23 (1959), 19-32.

[28] V. S. Vladimirov, Equations of Mathematical Physics, Nauka, Moscow 1967 (in Russian); English edition: Marcel Dekker, New York, 1971.

[29] V. S. Vladimirov, Methods of the Theory of Generalized Functions, Taylor \& Francis, London-New York, 2002.

[30] P. Wagner, Zur Faltung von Distributionen, Math. Ann. 276 (1987), 467-485.

[31] R. Wawak, Improper integrals of distributions, Studia Math. 86 (1987), 205-220.

[32] K. Yoshinaga, H. Ogata, On the convolutions, J. Sci. Hiroshima Univ. Ser. A 22 (1958), 15-24.

\author{
ANDRZEJ KAMIŃSKI \\ Institute of Mathematics, University of Rzeszów \\ ul. Rejtana 16A, 35-959 Rzeszów, Poland \\ E-mail: akaminsk@ur.edu.pl
}

\title{
Svetlana MincheVA-KamińsKa
}

Institute of Mathematics, University of Rzeszów

ul. Rejtana 16A, 35-959 Rzeszów, Poland

E-mail: minczewa@ur.edu.pl 